\title{
Feline patent Toxoplasma-like coccidiosis among feral cats (Felis catus) in Doha city, Qatar and its immediate surroundings
}

\author{
Marawan A. Abu-Madi ${ }^{*}$ and Jerzy M. Behnke ${ }^{2}$ \\ ${ }^{1}$ Department of Health Sciences, College of Arts and Sciences, Qatar University, P.O. Box 2713, Doha, Qatar; \\ ${ }^{2}$ School of Biology, University of Nottingham, University Park, Nottingham, NG7 2RD, U.K.
}

\begin{abstract}
Doha city has a high feral cat population and studies of hospital records in Doha have shown that human toxoplasmosis also occurs. Clearly, there is a need to understand the role of cats as vectors of human toxoplasmosis in the city and as a first step we assessed the extent of patent Toxoplasma-like coccidial infections among feral cats. Oocysts in cat faeces were detected between June 2008 and April 2010, from a range of locations radiating out of the city centre in concentric semi circular/elliptic rings and by north, west and south divisions within each of the rings. In total 4,652 cats were sampled and overall prevalence of oocysts was $9.1 \%$. Prevalence was $10.1 \%$ in the first summer, and then dropped to $8.4 \%$ in the following winter and further to $6.8 \%$ in the next summer before rising to $10.6 \%$ in the final winter of the study; this interaction between annual period and season was significant. There were also significant changes in prevalence across each of the consecutive months of the study, but no clear pattern was evident. Prevalence did not vary significantly by city sector and there was no difference in prevalence between the host sexes. We conclude therefore, that despite minor and significant perturbations, the prevalence of patent Toxoplasma-like coccidial infections among cats in Doha is remarkably stable throughout the year, across years and spatially within the city's districts.
\end{abstract}

\section{Keywords}

Feral cats, Doha, Qatar, coccidial oocysts, Toxoplasma gondii, toxoplasmosis, Hammondia, Besnoitia

\section{Introduction}

Doha city has an unusually high feral cat population that is estimated to outnumber its human inhabitants by $2-3: 1$, giving a total of approximately 2-3 million cats (Abu-Madi et al. 2008; personal communication from the Qatar Cat Control Unit [QCCU]). The animals were originally introduced into the city to control rodent populations, and although cats are not popular as pets in this city, the feral animals are popular and are tolerated because of this perceived benefit (Abu-Madi et al. 2010b; Elhag et al. 2002). Nevertheless, there is a control program to reduce the size of the cat population, implemented by the city authorities. Cats are trapped regularly in different locations throughout the city. Both sexes are neutered and any overtly sick animals are culled (Abu-Madi et al. 2010b).

In earlier work we described some of the helminth parasites harboured by these animals (Abu-Madi et al. 2010a) but the extent to which they act as reservoirs of protozoan para- sites is still largely unknown. However, hospital records show that human toxoplasmosis is quite widespread in the city, with up to $35 \%$ of women of child bearing age being reported to be seropositive (Abu-Madi et al. 2010b), and $41 \%$ of the elderly persons of both sexes in the population (Abu-Madi et al. 2008). Cats and their closely related felid relatives are the only known definitive hosts of Toxoplasma gondii (Tenter et al. 2000; Dubey 2009) but cats are also hosts to other closely related species of intestinal protozoa. For example, cats can harbour the coccidia of Hammondia spp. and Besnoitia spp. some of which cannot be distinguished on morphological or morphometric grounds from those of $T$. gondii, and may also harbour Isospora spp., Sarcocystis spp. and Cryptosporidium felis, which can be distinguished by morphology and size, and the Sarcomastigophoran (flagellate) Giardia felis (Bowman et al. 2002). Given the high density of cats in the city, it is clearly important to assess the prevalence of patent $T$. gondii-like coccidial infections among these animals as a first step towards 
eventually achieving a better understanding of their role in the transmission of human infectious disease of feline origin.

In this paper we report on the results of light microscopical examination of faecal samples for oocysts of $T$. gondiilike coccidial protozoa and for the first time we provide the prevalence figures for patent infections in the feral cat population of Doha city. Our objective was to obtain overall prevalence values for current patent infections among cats in the city, but also to assess how prevalence changes with season and time over successive years, and to establish whether prevalence is stable and hence predictable, or highly unstable in this urban feline population. Perhaps more importantly, we aimed to determine whether it is focused in particular locations either within the city boundaries or on the immediate periphery. As in most cities, housing standards vary enormously across the residential quarters of Doha. In some parts of the city, living quarters are densely packed, and these are principally populated by the immigrant workers (Elhag et al. 2002). Elsewhere housing is less dense and of a higher standard. These differences are likely to provide environments of varying suitability for feral rodent and cat populations. If prevalence varies spatially within the city, then efforts to control feline transmitted disease would benefit from more focused programs of control targeting those areas specifically rather than city-wide control as practiced at present. This would improve efforts to prevent disease transmission and might be significantly more cost-effective in the long-term.

\section{Materials and Methods}

\section{Location of sites}

Doha city is situated on the eastern edge of the Qatar peninsula, with the Al Corniche Lagoon as its eastern periphery (Fig. 1). Ring roads form concentric semi circles /ellipses radiating out from the city centre and these were used as guides for dividing the urban area and its immediate peripheral semi-urban environments into sectors for analysis of the distribution of infection among cats living in different parts of the city (the factor RING below). The innermost location was the city centre itself, then immediately adjacent to its outer periphery, ring 1 has its furthest edge on ring road $\mathrm{C}$. Ring 2 stretches from ring road $\mathrm{C}$ to ring road $\mathrm{E}$, and ring 3 represents the areas on the outside of ring road E, mainly outlying settlements which are semirural in character. Each of these 4 sectors was then divided further into a northern, western and southern sector (NWS SECTOR in the analysis below and see Fig. 1). There is no eastern sector because of the Al Corniche Lagoon. These sectors are listed in Table I, with examples of district names within each, and numbers of cats sampled, both by primary location and in total. Within each sector, traps were set out in places most likely to be frequented by cats at night, i.e. in alley ways, in back yards close to houses and near to rubbish bins and municipal garbage containers.

Table I. Locations at which cats were sampled and numbers of cats sampled in each (grand total $=4652)$

\begin{tabular}{|c|c|c|c|c|}
\hline $\begin{array}{l}\text { City centre } \\
\text { and ring }{ }^{1}\end{array}$ & $\begin{array}{l}\text { NWS } \\
\text { sectors }^{2}\end{array}$ & Districts & $\begin{array}{l}\text { Total by NWS } \\
\text { sectors }\end{array}$ & $\begin{array}{c}\text { Total by } \\
\text { ring }\end{array}$ \\
\hline City centre & North & Doha & 39 & \\
\hline City centre & West & Bin Mahoud & 21 & \\
\hline City centre & South & Al Mansourah, Al Muntazah, Najma & 171 & 231 \\
\hline Ring 1 & North & Daffna, Al Markhiya, Bin Omran, Madinat Khalifa & 900 & \\
\hline Ring 1 & West & Al Naser, Al Sadd, Hitmy & 70 & \\
\hline Ring 1 & South & Hilal & 312 & 1282 \\
\hline Ring 2 & North & Al Duhall, Al Gharafa, Al Zaghawa & 576 & \\
\hline Ring 2 & West & $\begin{array}{l}\text { Al Waab, Al Aziziya, Al Soudan, } \\
\text { Ain Khalid, Al Luqta, Maeither, } \\
\text { Morrah, Rayyan, Wajba }\end{array}$ & 1059 & \\
\hline Ring 2 & South & Mattar, Nuaija, Abu Hamour & 663 & 2298 \\
\hline Ring 3 & North & $\begin{array}{l}\text { Al Kaaban, Al Shamal, Al Kheraitiyat, Al Kheesa, } \\
\text { Al Khor, Simaisma, Umm Obairiya, Umm Qarn, } \\
\text { Umm Slal }\end{array}$ & 479 & \\
\hline Ring 3 & West & Al Sheehanya, Al Sailiya & 88 & \\
\hline Ring 3 & South & Al Wakra & 274 & 841 \\
\hline
\end{tabular}

${ }^{1}$ Rings stretch concentrically out of the centre of Doha, in semi circles from the north via the west to the south and with Doha's Al Coniche Lagoon to the east. Beginning with the city centre, ring 1 is the inner most ring around the city centre, and ring 3 is outermost. ${ }^{2}$ The rings were divided into northern, western and southern (NWS) sectors for each ring as shown in Fig. 1 


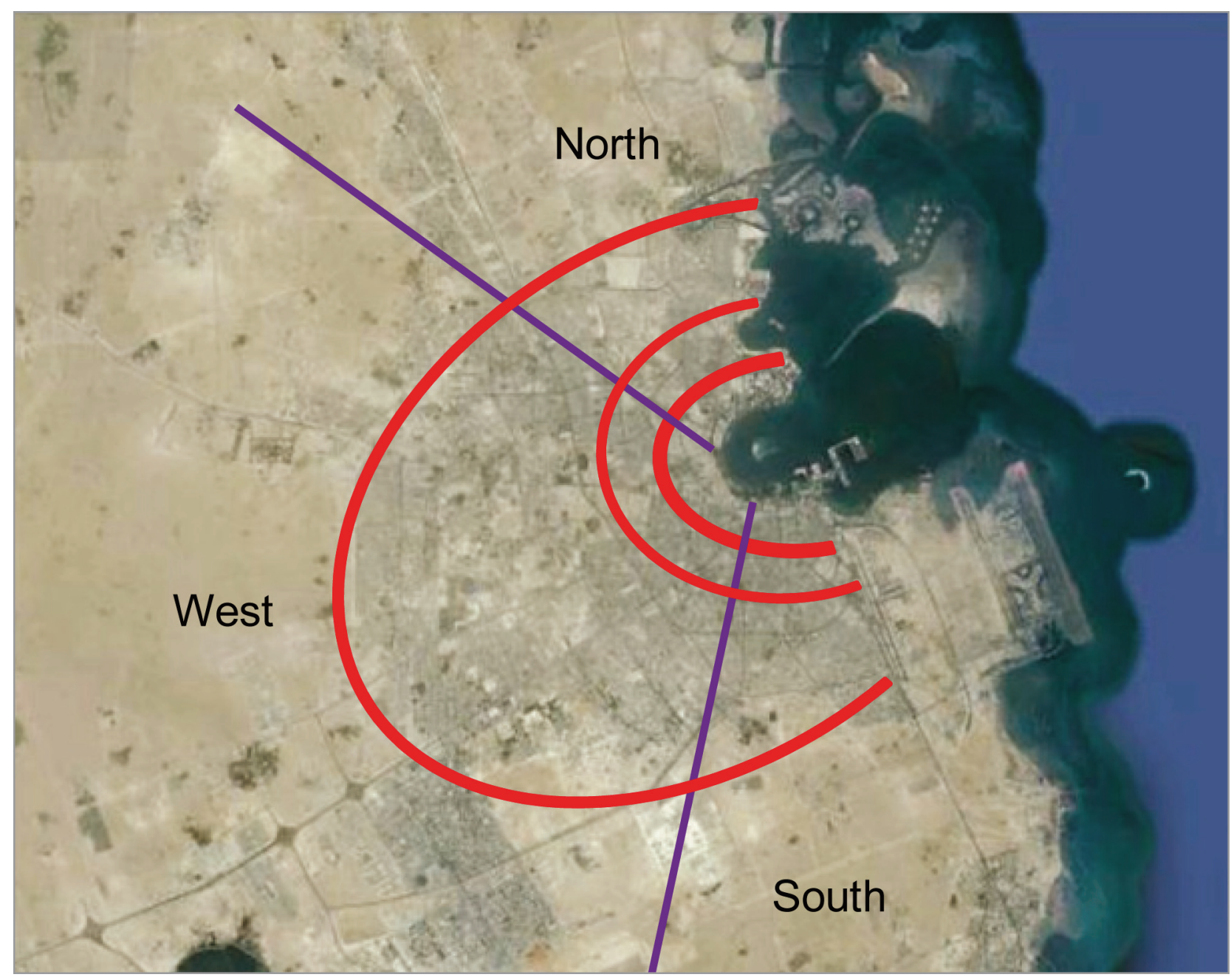

Fig. 1. Google Earth satellite image of Doha, showing also the approximate divisions of the city by rings and NWS sectors

\section{Cats}

Feral cats were caught live as part of the routine activities of the QCCU by well trained and experienced personnel. Traps, pre-baited with fish heads or canned cat food, were set out each evening, monitored during the night and replaced with fresh traps as necessary. Animals were retrieved from traps and assessed for sterilization status, age and pregnancy. Pregnant and lactating females, cats estimated to be less than 6 months old and sterilized cats were immediately released. Cats $\geq 6$ months old were eligible for the trap-neuter-return (TNR) program and were transported to a shelter for sterilization, respecting current animal welfare rules. To avoid re-sampling animals that had already been sampled and treated earlier, each animal was ear tagged with a small metallic tag on inspection. Each animal was sexed ('SEX' in the analysis), and the exact date on which it was examined was recorded. Qatar typically experiences two seasons ('SEASON' in the analysis). The summer season begins in early June, when daytime temperatures frequently exceed $45^{\circ} \mathrm{C}$ and seldom fall below $18^{\circ} \mathrm{C}$ at night, and lasts until the end of October with increasing hu- midity which peaks in August at $90 \%$, before subsiding to $70 \%$ towards the end of October. The winter season is considered to begin in early November and lasts until the end of May. During this period temperatures peak at about $28^{\circ} \mathrm{C}$ and may fall as low as $7^{\circ} \mathrm{C}$ at night and the mean humidity is usually in the range $75-86 \%$. The study began in June 2008, at the beginning of the summer and ended in April 2010 at the end of the second winter. In the analysis therefore, 'ANNUAL PERIOD' was used rather than calendar year because the former covered two complete sets of seasons; had the latter been applied, the continuity of seasons would have been disrupted within calendar years.

\section{Detection of Toxoplasma-like coccidial oocysts}

Stool examination was carried out in a safety cabinet, where each stool specimen was preserved in an ecofix preservative vial (Meridin Biosciences, Inc.). The contents were mixed vigorously by vortex and the homogenized stool sample was kept for half an hour at room temperature to ensure adequate fixation. The preserved specimen was mixed by vortex and fil- 
tered through a macro-con filtration unit for the removal of bulky debris. After filtration, 10\% formalin and ethyl acetate were added; the sample was centrifuged for $10 \mathrm{~min}$ at 3000 rpm and the fluid was discarded. The pellet was re-suspended by agitation, poured onto a microscope slide containing one drop of iodine and examined microscopically for the identification of Toxoplasma-like coccidial oocysts.

Under conventional light microscopical examination, the oocysts of $T$. gondii, Besnoitia darlingi and Hamondia hammondi cannot be distinguished on morphological and morphometric criteria, and therefore these formed a single taxon in the current project which we have referred to as Toxoplasma-like coccidia. The microscopists undertaking examination of the samples were experienced veterinary surgeons/technicians who were able to distinguish these oocysts from those of Isospora spp., Hammondia pardalis, Besnoitia wallacei, Cryptosporidium, the sporocysts of Sarcocystis spp. and the cysts of G. felis (Bowman et al. 2002).

\section{Statistical analysis}

Prevalence data (percentage of cats passing oocysts) are shown with $95 \%$ confidence limits (in columns in tables, in parenthesis in the text and as error bars on figures), calculated as described by Rohlf and Sokal (1995) employing bespoke software. All calculations were carried out on numbers based on three decimal places, but are rounded to the nearest single decimal place in the text and tables. Prevalence was analyzed by maximum likelihood techniques based on log linear analysis of contingency tables using the software package SPSS (Version 16.0.1.). Initially, a full factorial model was fitted, incorporating as factors SEX (2 levels, males and females), SEASON (2 levels, spring and winter), ANNUAL PERIOD ( 2 levels as given above), RING (4 levels, city centre and then rings 1, 2 and 3) and NWS SECTOR (3 levels, north, west and south sectors of each RING). INFECTION was considered as a binary factor (present/absent). The full factorial model comprising all possible interactions was simplified stepwise by the backward selection procedure with consecutive deletion of those combinations that did not contribute significantly to explaining variation in the data starting with the highest-level interaction. A minimum sufficient model was then obtained, for which the likelihood ratio of $\chi^{2}$ was not significant, indicating that the model was sufficient in explaining the data (these values are given in the legends to the figures as relevant). The importance of each term (i.e. interactions involving INFECTION) in the final model was assessed by the probability that its exclusion would alter the model significantly and these values relating to interactions that included presence/absence of infection are given in the text. The remaining terms in the final model that did not include presence/absence of infection are not given but can be made available from the authors on request. In some cases more restricted models were fitted to test specific hypotheses (e.g. MONTH at 22 levels; note no data were available for month 16, May 2009), as explained later in the text.

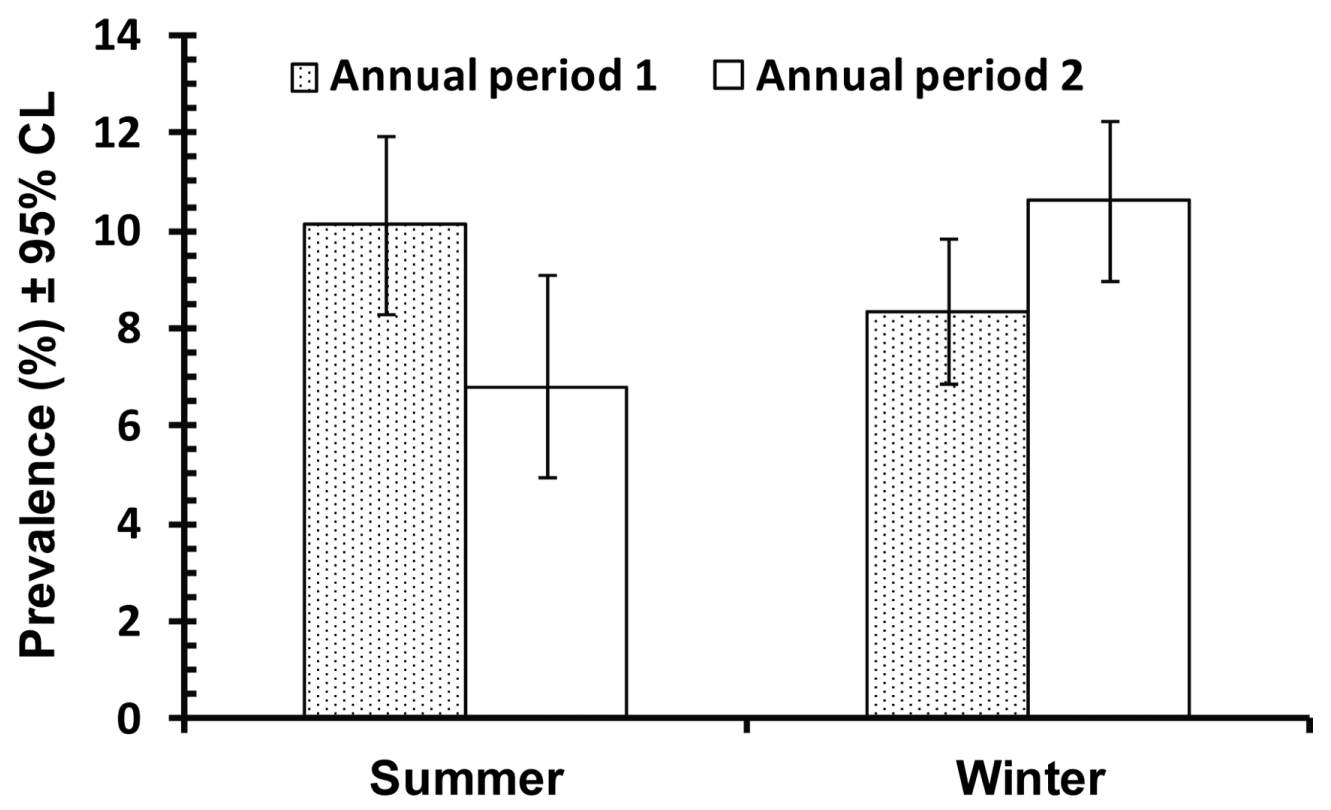

\section{Season}

Fig. 2. Variation in prevalence of Toxoplasma-like oocysts in faecal samples of cats across two seasons and two consecutive annual periods. The statistical analysis is given in the text. The minimum sufficient model (for goodness of fit of this model, likelihood ratio $\chi_{125}^{2}=139$, $P=0.18$ ) included four terms of which only one carried an interaction with presence/absence of oocysts and this is illustrated in the figure 


\section{Results}

\section{Sample size and overall prevalence of infection}

A total of 4,652 cats was examined and of these 1048 were female cats and 3604 were males. The numbers of cats examined in 2008, 2009 and 2010 were 1531, 2157 and 964 respectively, but because seasons did not conveniently fall across calendar years, the effect of time was analysed by annual periods. The numbers of cats in the first annual period (first summer-winter cycle) were 2395 and in the second 2257. In the summer periods 1928 cats were sampled, whilst in winter the number was 2724 . Of the 4652 cats examined, 425 (prevalence $=9.1 \%$ [8.31-10.1]) were found to be passing Toxoplasma-like oocysts.

\section{Factors affecting prevalence}

Statistical analysis revealed only one significant interaction. Prevalence of oocysts varied between the seasons but this variation was not consistent across the two annual periods studied (Fig. 2; for SEASON*ANNUAL PERIOD*INFECTION, $\left.\chi_{1}^{2}=11.0, P=0.001\right)$. In the first annual period, which stretched from June 2008 until May 2009, prevalence of oocysts dropped from summer to winter, but in the next annual period the pattern was reversed. However, the extent of the actual changes was not marked, with the lowest value recorded in the summer of the second annual period at $6.7 \%$, and the highest in the following winter at $10.6 \%$, representing an in- crease of 3.8\%. Although in relative terms, this was an increase of $56.7 \%$, biologically this was probably of little consequence.

\section{Monthly changes over time}

Fig. 3 shows monthly prevalence from June 2008 to May 2010. The lowest mean was $4.0 \%$ in month 22 (October 2009) and the highest was 16\% in month 27 (March 2010). Analysis of these data, in the absence of other factors, indicated that there were significant fluctuations from month to month (for MONTH*INFECTION, $\chi_{23}^{2}=39.8, P=0.016$ ). The effect remained significant when RING and NWS SECTORS were included as factors, and also when initial full factorials models were fitted with ANNUAL PERIOD and SEASON as factors. Although, there is no strong overall pattern to the monthly changes, curve fitting procedure in SPSS indicated that the data were best described by the quadratic equation $y=17.39$ $1.144 x+0.034 x^{2}$, which generates a shallow concave curve that dips in the middle of the graph (as shown on Fig. 3). While this was the best-fit curve $\left(R^{2}=0.204\right)$, it nevertheless did not represent a significant relationship $\left(F_{2,19}=2.43, P=0.115\right)$.

\section{Variation in prevalence across locations in the city}

The prevalence rates for different sectors of the city are summarized in Table II. These range from zero in the west of the city centre to $20.5 \%$ in the north of the city centre. However, in relation to other sectors, relatively few cats were sampled

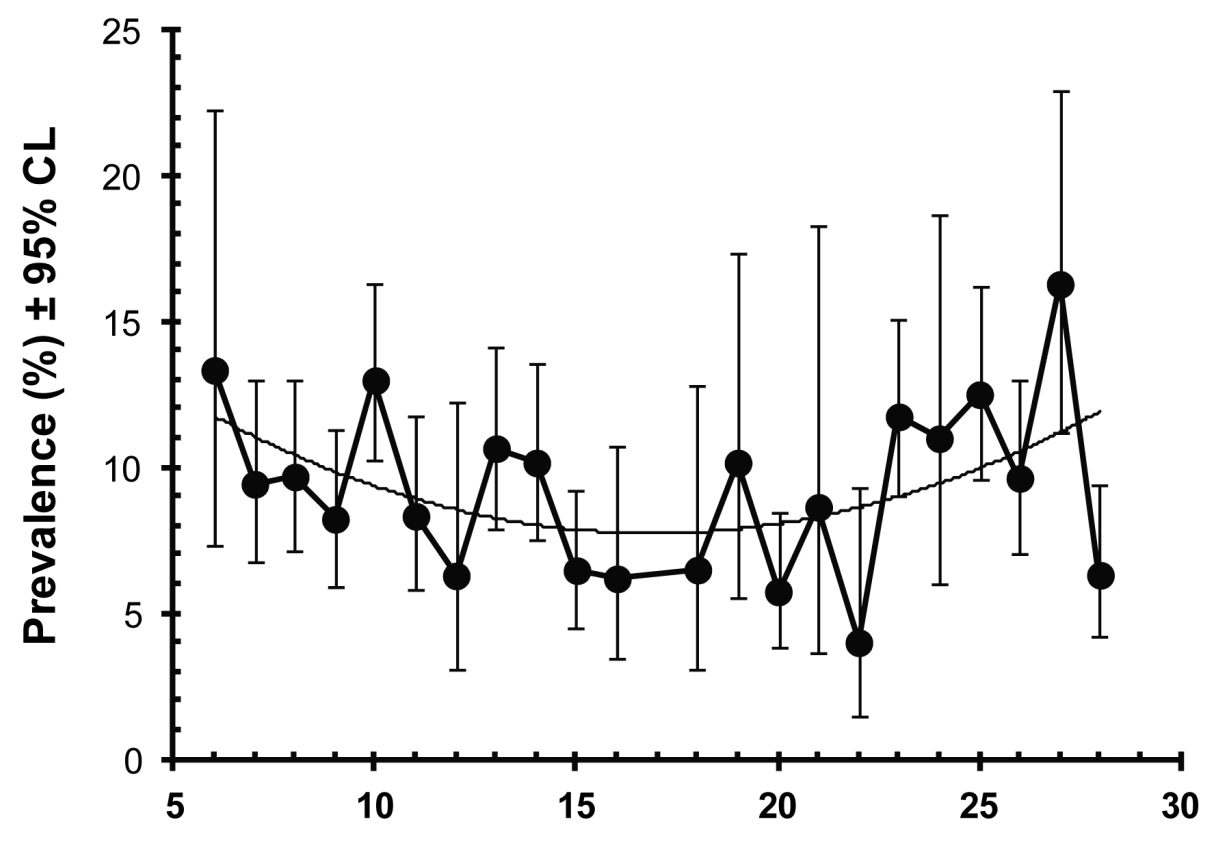

\section{Month}

Fig. 3. Changes in prevalence of Toxoplasma-like oocysts in faecal samples of cats as recorded monthly over the period from June 2008 (month 6 on the $x$-axis) until May 2010 (month 28), with the line of best-fit $\left(y=17.39-1.144 x+0.034 x^{2}\right)$ 
Table II. Prevalence of Toxoplasma-like coccidial oocysts in cats from different sectors of Doha city

\begin{tabular}{lccc}
\hline Ring & $\begin{array}{c}\text { NWS } \\
\text { sectors }\end{array}$ & Prevalence \% & 95\% Confidence limits \\
\hline City centre & North & 20.5 & $10.34-36.31$ \\
City centre & West & 0 & $0-15.89$ \\
City centre & South & 9.4 & $5.18-16.03$ \\
& & & \\
Ring 1 & North & 9.1 & $6.98-11.80$ \\
Ring 1 & West & 10.0 & $4.82-19.14$ \\
Ring 1 & South & 9.3 & $6.59-12.95$ \\
& & & \\
Ring 2 & North & 10.2 & $8.37-12.43$ \\
Ring 2 & West & 7.9 & $6.33-9.82$ \\
Ring 2 & South & 8.8 & $6.93-10.96$ \\
Ring 3 & & & \\
Ring 3 & North & 11.5 & $7.69-16.53$ \\
Ring 3 & West & 8.0 & $2.99-17.91$ \\
\hline
\end{tabular}

from either of these two locations. Excluding the city centre, prevalence was remarkably constant across all other sectors, varying only from 7.3 to $11.5 \%$. There was an indication from the data that cats from the northern sectors may have a higher prevalence. In the city centre, in rings 2 and 3, prevalence was numerically higher. When pooled across rings and expressed only on the basis of NWS SECTORs, the values for prevalence were $10.2 \%$ (8.90-11.56), 7.9\% (6.43-9.65) and $8.7 \%(7.20-10.12)$ for north, west and south sectors, respectively, but when tested in the absence of other factors NWS SECTOR approached but did not reach statistical significance (for NWS SECTOR*INFECTION $\chi_{4652}^{2}=5.49, P$ $=0.064)$.

\section{Variation in prevalence between the sexes}

Prevalence was nominally, but not significantly, higher among male $(9.4 \%$ [8.43-10.33]) compared with female cats $(8.3 \%$ [6.65-10.24]).

\section{Discussion}

This study has drawn attention to the remarkable stability of the prevalence of patent Toxoplasma-like coccidial infections among feral cats in Doha. Dubey et al. (2010) encouraged further studies to assess the diversity and transmission of $T$. gondii in animals in the region and our study has contributed to this objective. The overall prevalence of oocysts in faeces was $9.1 \%$ with most monthly figures varying by just $\pm 4 \%$, the occasional extremes being $4 \%$ in month 22 and $16 \%$ in month 27 , but otherwise showing no significant trend in prevalence over time. However, our results emphasize the atypically high prevalence of faecal oocyst shedding among adult cats compared to findings from previous studies for T. gondii (Dabritz and Conrad 2010).
At this stage the exact identity of the oocysts in the present study is not absolutely certain. Purely on the basis of morphology and morphometrics they may have represented a combination of $H$. hammondi, B. wallacei and T. gondii. However, $B$. wallacei can be largely eliminated since despite a single report of its presence in Kenya (Ng'ang'a et al. 1994), the parasite is predominantly restricted to Australia, New Zealand and some Pacific Islands (Bowman et al. 2002). Similarly, $H$. pardalis which has considerably bigger oocysts than $T$. gondii is only found in C. America and the southern parts of N. America (Bowman et al. 2002). Therefore, in view of the above the most likely concurrent infection with $T$. gondii in our cats was H. hammondi which has a global distribution but does not cause disease in cats and is unlikely to be transmissible to humans (Bowman et al. 2002). However, Bowman et al. (2002) reported that the prevalence of oocysts with this species is extremely low in cats in all published studies, and to the best of our knowledge there have been no reports of its presence in the Middle East, so even if present it is likely to be a minor contaminant in our data, but nevertheless, erring on the side of caution we have referred throughout to Toxoplasma-like coccidial infections.

Although there was some variation in prevalence of patent infections in our study, which was reflected in changes, for example, between the seasons of successive years and consecutive months, these were relatively minor and did not conform to any evident cycles or long-term trends, across the study period. There were no changes in prevalence between months that we could link to any underlying biological phenomena that might help to identify the risk of transmission to the human population.

However, whatever temporal differences in prevalence may have been observed, and with the exception of the city centre sectors, the prevalence of infection across different outlying sectors of Doha city showed remarkably little variation. Cats from the various districts of Doha, both in terms of rela- 
tive distance to the city centre and whether they were in the northern, western, or southern regions of the study area, all showed similar rates of prevalence. Cats are usually considered to be territorial animals that defend their territories vigorously (Liberg 1980; Horn et al. 2011), so we might have expected to see infections aggregating in particular locations within the city, perhaps in particular territories. This was clearly not the case, at least at the district level at which this study was conducted and hence, our results do not encourage us to believe that control of toxoplasmosis should focus on any particular locale. There appear to be no foci of infection where prevalence is considerably higher than elsewhere, that might have acted as significant reservoirs, seeding transmission to other districts in the city. For these reasons, therefore, refinement of current control programs by implementation of more selective trapping and control is not warranted.

Transmission of $T$. gondii can be direct from cats to other animals (including other cats) via faecal shedding of the oocysts; however, as with $H$. hammondi the parasite also exploits intermediate hosts, such as rodents. Wild rats are plentiful in Doha city and have been shown previously to carry a significant parasite burdens although they have not been assessed yet for T. gondii infection (Abu-Madi et al. 2001). Rodents also have territories and may play a role locally in spreading infection but are unlikely to be freely intermingling on a regular basis across the whole city. Wild birds can also act as hosts for $T$. gondii, although not H. hammondi, and are another potential source of infection for cats, as they forage throughout the city for food and have a greater potential for disseminating infection over longer distances. However, the extent to which the local feral cat population preys on birds and the role of birds in transmission of $T$. gondii locally have not been assessed.

Our overall figure of $9.1 \%$ prevalence of patent Toxoplasma-like coccidial infections among adult cats is markedly higher than that cited for $T$. gondii in other reports that have used coproscopical methods (Schares et al. 2008; reviewed by Dabritz and Conrad 2010). However, it is pertinent that Dubey et al. (2010) have reported recently on the high prevalence of antibodies to $T$. gondii (49/57 tested positive) in wild felids in the UAE, and at a wildlife preservation institute in Qatar (21/27 were seropositive). Moreover, following the development of highly sensitive polymerase chain reaction (PCR) primers specific for $T$. gondii DNA from oocysts, Salant et al. (2007) reported a prevalence almost identical to our data $(9.0 \%)$ among stray cats in Jerusalem. Despite the difference in methodology and their relative sensitivities, these data show that there is a precedent for prevalence values of $T$. gondii in feral cats that is as high as $9 \%$. Nevertheless, our results are all the more surprising since we did not sample young cats less than 6 months old, which would have been even more susceptible to infection and among which prevalence would have been expected to have been even higher.

In general, cats shed the oocysts of both $T$. gondii and $H$. hammondi for only a brief period after initial infection; al- though infrequent, secondary periods of shedding have occasionally been observed in the case of $T$. gondii. It is believed these subsequent periods of shedding arise either when animals experience a subsequent high challenge with infective oocysts or during periods of immunodepression from stress or other infections (Tenter et al. 2000). It is possible that feral cats living in the harsh environmental conditions in Doha city, with its extreme summer temperatures and marked aridity, perhaps combined with exposure to other feline pathogens, were less able to resist infection compared with urban feline populations in more moderate climates. In this case we might have expected to see a higher prevalence during summer, but such a pattern was not evident in our data, since in the first summer prevalence was relatively high, but in the following summer it was lower than in the intervening winter. This is clearly an important issue to resolve in future work.

There is an established cat control program in Doha city and it is possible that culling of the infected animals is helping to reduce human T. gondii infection levels throughout the city. In the past cats that were seropositive for T. gondii were culled, although this practice was abandoned in 2005 and presently, cats that are positive on the basis of presence of faecal $T$. gondii-like oocysts are culled by the QCCU. To set this in context, from March 2006 to June 2009, 24,372 cats were trapped by the QCCU, of which 2,653 were culled (10.9\%), 9,637 were spayed/neutered and these latter, together with the rest, were then released (QCCU, personal communication). However, extrapolating from the current study's figure of $9.1 \%$ of cats actively disseminating coccidial oocysts to the whole population, the percentage of cats with patent infections culled over that period was less than $2 \%$ of the whole population. Therefore, the extent to which the culling of infected animals actually impinges on overall transmission efficiency of $T$. gondi and its close relative, $H$. hammondi, and thereby affects overall prevalence levels, is probably marginal and not a major factor. Moreover, the release of animals less than 6 months of age, which would be expected to be more susceptible to infection than adult animals, clearly leaves a large pool of potential reservoirs of infection to disseminate oocysts and perpetuate the persistence of the parasite in the population. The role of culling as a measure for keeping overall prevalence down could only be convincingly established by comparing prevalence in districts where infected cats are culled with those in which they are not, but such a change (i.e. cessation of culling in some districts to provide control sites) to the established feline control program is unlikely to be adopted.

Although, at this stage we cannot discount $H$. hammondi contributing significantly to the $T$. gondii-like cysts, we believe that there is no room for complacency because $T$. gondii poses a significant human health risk: it can have particularly devastating effects on the unborn and on children that then suffer lifelong consequences (Hill and Dubey 2002; Montoya and Liesenfeld 2004). Cats carrying patent infections shed hundreds of millions of oocysts even if for only for a brief period of time (Tenter et al. 2000), and feral animals pose a clear threat 
for the spread of disease. Transmission of $T$. gondii can be also through environmental contamination, or through contamination of food which enters the human food chain, particularly since feral cats are allowed to roam unmolested in the city, can often be observed foraging in dustbins on discarded food and enter food halls where food is prepared, processed and sold (Pers. obs.). Further work is needed to clearly identify the proportion of cats actually infected with $T$. gondii rather than $H$. hammondi, and this can be done by inoculation into mice, followed by relevant histological analysis and by molecular methods, specific diagnostic PCR primers having been developed for both species and used in recent studies (Homan et al. 2000; Salant et al. 2007; Schares et al. 2008). If T. gondii proves to be largely responsible for the oocysts in our study it will be important in future to minimise $T$. gondii transmission from feral cats to the human population in Doha. Ideally, the feral cat population should be reduced further, but it seems unlikely this will happen because of the popularity of feral cats among the inhabitants, and the belief that they play a vital role in rodent control. The situation is further confounded by the legal importation of approximately 1000-2000 cats annually, mostly by expatriate owners who keep their pets indoors. Others are imported mainly from Egypt by pet marketers for sale in Qatar and are also generally kept indoors, but about $75 \%$ of these latter have been found to be positive for Toxoplasma-like oocysts in faeces by the QCCU. The extent of illegal importation of cats is not known.

Finally, although our study has raised interesting and pertinent questions which we hope to resolve through further work in the years ahead, data from the current study should help to inform the authorities on the role of cats as hosts of zoonotic protozoan infections and to assist them in developing future strategies to address the health and management of the feral cat population in Doha. Ultimately this should benefit the human inhabitants of this city as well.

Acknowledgments. We wish to express our sincere and grateful thanks to Qatar National Research Funds for generous financial support through Student grant UREP $05-060-3-019$ and to the Department of Animal Resources, Ministry of Environment, Doha, Qatar for providing samples used in the study.

\section{References}

Abu-Madi M.A., Al-Molawi N., Behnke J.M. 2008. Seroprevalence and epidemiological correlates of Toxoplasma gondii infections among patients referred for hospital-based serological testing in Doha, Qatar. Parasites and Vectors, 1, 39.

Abu-Madi M.A., Behnke J.M., Prabhaker K.S., Al-Ibrahim R., Lewis J.W. 2010a. Intestinal helminths of feral cat populations from urban and suburban districts of Qatar. Veterinary Parasitology, 168, 284-292.

Received: June 13, 2013

Revised: November 23, 2013

Accepted for publication: April 4, 2014
Abu-Madi M.A., Behnke J.M., Dabritz H.A. 2010b. Toxoplasma gondii seropositivity and co-infection with TORCH pathogens in high-risk patients from Qatar. American Journal of Tropical Medicine and Hygiene, 82, 626-633.

Abu-Madi M.A., Lewis J.W., Mikhail M., El-Nagger M.E., Behnke J.M. 2001. Monospecific helminth and arthropod infections in an urban population of brown rats from Doha, Qatar. Journal of Helminthology, 75, 313-320.

Bowman D.D., Hendrix C.M., Lindsay D.S., Barr S.C. 2002. Feline Clinical Parasitology. Iowa State University Press, Iowa, U.S.A.

Dabritz H.A., Conrad P.A. 2010. Cats and Toxoplasma: implications for public health. Zoonoses and Public Health, 57, 34-52.

Dubey J.P. 2009. History of the discovery of the life cycle of Toxoplasma gondii. International Journal for Parasitology, 39, 877-882.

Dubey J.P., Pas A., Rajendran C., Kwok O.C.H., Ferreira L.R., Martins J., Hebel C., Hammer S., Su C. 2010. Toxoplasmosis in sand cats (Felis margarita) and other animals in the breeding centre for endangered Arabian wildlife in the Unites Arab Emirates and Al Wabra Wildlife Preservation, the state of Qatar. Veterinary Parasitology, 172, 195-203.

Elhag E.A., Aspinall S., Schwarze H., Boer B., Osman A. 2002. Environmental survey in Abu-Hamour, Doha, Qatar. UNESCO Report for the Ministry of Municipal Affairs and Agriculture. Doha, Qatar : UNESCO .

Hill D., Dubey J.P. 2002. Toxoplasma gondii: transmission, diagnosis and prevention. Clinical Microbiology and Infection, 8, 634-640.

Homan W.L., Vercammen M., de Braekeleer J., Verschueren H. 2000. Identification of a 200- to 300-fold repetitive 529 bp DNA fragment in Toxoplasma gondii, and its use for diagnostic and quantitative PCR. International Journal for Parasitology, 30, 69-75.

Horn J.A., Mateus-Pinilla N., Warner R.E., Heske E.J. 2011. Home range, habitat use, and activity patterns of free-roaming domestic cats. The Journal of Wildlife Management, 75, 11771185.

Liberg O. 1980. Spacing patterns in a population of rural free roaming domestic cats. Oikos, 35, 336-349.

Montoya J.G., Liesenfeld O. 2004. Toxoplasmosis. Lancet, 363 , 1965-1976.

Ng'ang'a C.J., Kanyari P.W., Munyua W.K. 1994. Isolation of Besnooitia wallacei in Kenya. Veterinary Parasitology, 52, 203-206.

Rohlf F.J., Sokal R.R. 1995. Statistical Tables (3 ${ }^{\text {rd }}$ Edition) W.H. Freeman and Company, San Francisco.

Salant H., Markovics A., Spira D.T., Hamburger J. 2007. The development of a molecular approach for coprodiagnosis of Toxoplasma gondii. Veterinary Parasitology, 146, 214-220.

Schares G., Globokar Vrhovec M., Pantchev N., Herrmann D.C., Conraths F.J. 2008. Occurrence of Toxoplasma gondii and $\mathrm{Ha}$ mondia hammondi oocysts in the faeces of cats from Germany and other European countries. Veterinary Parasitology, 152, 34-45.

Tenter A.M., Heckeroth A.R., Weiss L.M. 2000. Toxoplasma gondii: from animals to humans. International Journal for Parasitology, 30, 1217-1258. 